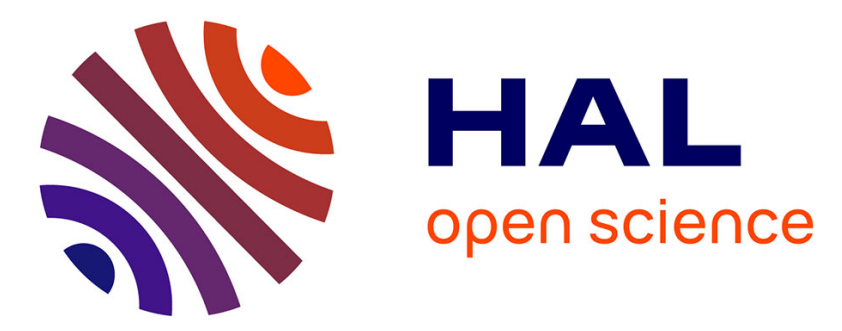

\title{
A polynomial chaos approach to the robust analysis of the dynamic behaviour of friction systems
}

\author{
Lyes Nechak, Sébastien Berger, Evelyne Aubry
}

\section{To cite this version:}

Lyes Nechak, Sébastien Berger, Evelyne Aubry. A polynomial chaos approach to the robust analysis of the dynamic behaviour of friction systems. European Journal of Mechanics - A/Solids, 2011, 30, pp.594-607. 10.1016/j.euromechsol.2011.03.002 . hal-00851925

\section{HAL Id: hal-00851925 \\ https://hal.science/hal-00851925}

Submitted on 19 Aug 2013

HAL is a multi-disciplinary open access archive for the deposit and dissemination of scientific research documents, whether they are published or not. The documents may come from teaching and research institutions in France or abroad, or from public or private research centers.
L'archive ouverte pluridisciplinaire HAL, est destinée au dépôt et à la diffusion de documents scientifiques de niveau recherche, publiés ou non, émanant des établissements d'enseignement et de recherche français ou étrangers, des laboratoires publics ou privés. 


\title{
A Polynomial Chaos Approach to the Robust Analysis of the
}

\section{Dynamic Behaviour of Friction Systems}

Lyes nechak ${ }^{\mathrm{a}}$, Sébastien Berger ${ }^{\mathrm{a}}$, Evelyne Aubry ${ }^{\mathrm{a}}$

a MIPS Laboratory, University of Haute Alsace, 12 Rue des frères Lumière 68093

Mulhouse, France, lyes.nechak@uha.fr sebastien.berger@uha.fr evelyne.aubry@uha.fr, Phone : 0389336944, Fax :0389423282

Corresponding author : Sébastien Berger

\begin{abstract}
This paper presents a dynamic behaviour study of non-linear friction systems subject to uncertain friction laws. The main aspects are the analysis of the stability and the associated non-linear amplitude around the steady-state equilibrium. As friction systems are highly sensitive to the dispersion of friction laws, it is necessary to take into account the uncertainty of the friction coefficient to obtain stability intervals and to estimate the extreme magnitudes of oscillations. Intrusive and nonintrusive methods based on the polynomial chaos theory are proposed to tackle these problems. The efficiency of these methods is investigated in a two degree of freedom system representing a drum brake system. The proposed methods prove to be interesting alternatives to the classic method such as parametric studies and Monte Carlo based techniques.
\end{abstract}

Key word: Dry friction systems, Nonlinear dynamic systems, stability, limit cycle, robustness, uncertainty propagation, polynomial chaos, Lyapunov function, SOS programming.

\section{Introduction}

Dry friction systems play a key role in numerous industrial applications such as braking, wiping and clutch systems (Sinou and Jezquel, 2007). The analysis of stability and dynamic behaviour associated with this particular class of nonlinear dynamic systems is of major importance (Sinou et al, 2004).

Several parametric studies have shown the great sensitivity of the dynamic behaviour of friction systems to design parameters, in particular to friction laws (Ibrahim, 1994a, 1994b ; Hoffman and 
Gaul, 2003; Shin et al, 2002a, 2002b; Sinou et al, 2006a, 2006b; Sinou and Jezequel, 2007). However, the friction coefficient admits strong dispersions (Chevennement et al, 2005, 2007). Therefore, it becomes necessary to take into account these uncertainties in order to ensure the robustness of the analysis. A robust study of non-linear dynamic behaviour will help to analyze stability and to predict the vibration levels according to the parameters variability. Several methods are proposed in the literature. They are called "methods of uncertainty propagation" in which the probabilistic approach occupies a dominating place. Monte Carlo (MC) simulation is a well known technique in this field (Fishman, 1996). It can give the entire probability density function of any system variable, but it is often too costly since a great number of samples are required for reasonable accuracy. Parallel simulation (Papadrakakis and Papadopoulos, 1999), Latin Hypercube Sampling (Helton and Davis, 2003) and proper orthogonal decomposition (Lindsley and Beran, 2005) are some solutions proposed to circumvent the computational difficulties of the MC method.

Polynomial chaos expansion (PCE) is presented in the literature as a more efficient probabilistic tool for uncertainty propagation. It was first introduced by Wiener. (1938) and pioneered by Ghanem and Spanos. (1991) who used Hermite orthogonal polynomials to model stochastic processes with Gaussian random variables. The exponential convergence of such expansion has been shown by Cameron and Martin. (1947) and generalized to various continuous and discrete distributions using orthogonal polynomials from the so called Askey-scheme (Askey and Wilson, 1985; Xiu and Karniadakis, 2003; Wang and Karniadakis, 2006).

Polynomial chaos (PC) gives a mathematical framework to separate the stochastic components of a system response from the deterministic ones. The stochastic Galerkin method (Babuska et. al, 2004; Ghanem and Spanos, 1991; Le Maitre et. Al, 2009), collocation and regression methods (Babuska et.al, 2007; Crestaux et. al, 2009) are used to compute the deterministic components called stochastic modes in an intrusive and non intrusive manner while random components are concentrated in the polynomial basis used. Non intrusive procedures prove to be more advantageous for stochastic dynamic systems since they need no modifications of the system model, contrary to the intrusive method. In the latter, Galerkin techniques are used to generate a set of deterministic coupled equations from the stochastic system model, then a suitable algorithm is adapted to obtain stochastic modes. 
The capabilities of polynomial chaos expansion have been tested in numerous applications, such as treating uncertainties in environmental and biological problems (Isukapalli and Georgopoulos, 1998a, 1998b) and in multibody dynamic systems (Sandu et al, 2006a, 2006b), solving ordinary and partial differential equations (Williams, 2006; Xiu and Karniadakis, 2002, 2003), sensitivity analysis (Crestaux et. al, 2009; Sudret, 2007), parameter estimation (Saad and al, 2007; Blanchard et. al, 2009, 2010a, 2010b; Smith et. al, 2007) and controller design problems, (Hover et. al, 2006; Nagy and Bratz, 2006). In the particular case of the problem dealt with in this paper, recent research work has been carried out. Indeed, the intrusive approach is a new one to analyze the stability of uncertain dynamic systems (Fischer and Bhattacharya, 2008). Fisher analyzes the stability of equilibrium in stochastic linear and polynomial dynamic systems by checking - with direct Lyapunov approach - the stability of the deterministic dynamic systems resulting from the application of intrusive polynomial chaos. Polynomial chaos has also been proposed recently for limit cycle oscillations (LCOs). Beran and coauthors consider in particular uncertain aerodynamic systems. In this study, the authors use the Wiener-Hermite polynomial expansion to analyze the variability of the limit cycle oscillations of an aerodynamic system according to Gaussian uncertainties in physical parameters, (Beran et al, 2006) . The same problem is dealt with in other studies (Millman et al, 2003; Pettit and Beran, 2006; Witteveen et. al, 2007). All come to the same conclusion: polynomial chaos gives good approximations of short term statistics (in terms of mean value and variance of LCO amplitudes) but presents a number of difficulties and limits to well approximate long term statistics.

In the particular case of friction systems, there are only few studies on this class of systems and no applications with polynomial chaos have been recorded. For instance, in the study of Ragot and coauthors, the interval theory is used to surround the dispersion of limit cycle oscillations due to the uncertainty of the friction coefficient (Ragot et. al, 2008); an overestimation problem is revealed which is due to accumulations of errors in time leading to the divergence of the envelopes of limit cycle amplitudes.

The main originality of the present paper is that the uncertainty of the friction coefficient in the dynamic behaviour study of friction systems is taken into account. The main objective is to investigate 
the capabilities of the polynomial chaos approach concerning the stability and limit cycle of this important class of nonlinear dynamic systems subject to uncertain friction laws. So, a two-degree of freedom system modelling the dynamic behaviour of a drum brake is considered (Hultèn, 1993; Sinou and Jezquel, 2007). The theoretical basis of both the polynomial chaos and the Lyapunov approach are presented in Section 2. Intrusive and non-intrusive methods are detailed as well as how to search for a Lyapunov function using sum of square polynomials. Section 3 is devoted to the study of the dynamic behaviour of the two degree of freedom friction system. The system is first presented, its stability is investigated and the LCO dispersion is estimated. Conclusions and perspectives are given at the end of the paper.

\section{Theoretical methods}

\subsection{Polynomial chaos}

Polynomial chaos establishes a separation between the stochastic components of a random function and its deterministic components. Here is the mathematical framework of this approach. From the Wiener theory and the generalized Cameron-Martin theorem, any second order random process $x$ can be expanded in a convergent (in the mean square sense) polynomial function series as:

$$
x=\sum_{j=0}^{\infty} \bar{x}_{j} \phi_{j}(\xi)
$$

$\xi$ is a vector of $d$ independent random variables with a known joint density function $W(\xi), \bar{x}_{j}$ are the stochastic modes of the random process $x$ and $\phi_{j}$ are orthogonal polynomial functions satisfying the following relations:

$$
\begin{aligned}
\left\langle\phi_{i}, \phi_{j}\right\rangle & =\int \phi_{i}(\xi) \phi_{j}(\xi) W(\xi) d \xi \\
& = \begin{cases}0 & i \neq j \\
\left\langle\phi_{i}^{2}\right\rangle & i=j\end{cases}
\end{aligned}
$$

$\langle\square$ being the internal product operator. 
In practice, polynomial chaos expansion is truncated to a finite number of terms $P$ which is demonstrated to be dependent on the polynomial chaos order $r$ and stochastic dimension $d$ denoting the number of uncertain parameters.

$$
x \approx \sum_{j=0}^{P} \bar{x}_{j} \phi_{j}(\xi)
$$

and

$$
P=((d+r) ! / d ! r !)-1
$$

Then, computing $x$ is turned into the problem of finding the coefficients $\bar{x}_{j}$ of its truncated expansion. Two main approaches are defined to calculate these coefficients called stochastic modes. The non-intrusive approach includes some techniques (non-intrusive spectral projection (NISP), regression) which use simulations corresponding to particular samples of the random parameters. The principal advantage of these techniques is related to the fact that no modification is performed on the system model contrary to the intrusive approach which needs the Galerkin techniques to generate a set of coupled deterministic equations from the uncertain system model. To explain how to use intrusive and nonintrusive techniques, consider the class of polynomial dynamic systems with a control parameter $\mu$, described by the following smooth vector field:

$$
\begin{aligned}
f: \mathfrak{R}^{n} \times \mathfrak{R} & \rightarrow \mathfrak{R}^{n} \\
& (x, \mu) \rightarrow f(x, \mu)
\end{aligned}
$$

such that $\quad \dot{x}(t)=f(x(t), \mu)$

Equivalently:

$$
\dot{x}_{i}(t)=f_{i}\left(x_{1}(t), \ldots, x_{n}(t), \mu\right), \quad i=1, \ldots, n
$$

Note that choice of polynomial dynamic systems is not restrictive since any smooth nonlinear function can be approximated by a polynomial function, using Taylor's series expansion.

Consider a uniform distribution for parameter $\mu$. As a consequence, the state variables also become uncertain. According to the Askey scheme (Askey and Wilson, 1985), Legendre polynomials $L_{j}(\xi)$ are more suitable for uniform uncertainties. So, all random variables can be expanded in terms of 
Legendre polynomials. As the control parameter $\mu$ is a constant, the first order Wiener-Legendre expansion is sufficient to describe it while the random state variables can be approximated by:

$$
x_{i}(t, \xi) \approx \sum_{j=0}^{P} \bar{x}_{i, j}(t) L_{j}(\xi), i=1, \ldots, n
$$

where $\xi \in[-1,1]$ is the new stochastic variable uniformly distributed on $[-1,1]$ which is the orthogonality interval of the Legendre polynomials.

Stochastic modes $\bar{x}_{i, j}(t)$ are then computed, using the intrusive or non-intrusive spectral projection or regression methods.

\subsubsection{Intrusive approach}

The intrusive approach can be summarized in four steps.

1. Substitute the truncated Wiener Legendre expansion (7) in the system equations (6).

$$
\sum_{j=0}^{P} \dot{\bar{x}}_{i, j}(t) L_{j}(\xi)=f_{i}\left(\sum_{j=0}^{P} \bar{x}_{1, j}(t) L_{j}(\xi), \ldots, \sum_{j=0}^{P} \bar{x}_{n, j}(t) L_{j}(\xi), \mu(\xi)\right), i=1, \ldots, n
$$

2. Compute the Galerkin projection as follows:

$$
\begin{aligned}
& \left\langle\sum_{j=0}^{P} \dot{\bar{x}}_{i, j}(t) L_{j}(\xi), L_{l}(\xi)\right\rangle= \\
& \quad\left\langle f_{i}\left(\sum_{j=0}^{P} \bar{x}_{1, j}(t) L_{j}(\xi), \ldots, \sum_{j=0}^{P} \bar{x}_{n, j}(t) L_{j}(\xi), \mu(\xi)\right), L_{l}(\xi)\right\rangle, i=1, \ldots, n, l=0, \ldots, P
\end{aligned}
$$

Using the orthogonal property of Legendre polynomials, a system of $P+1$ deterministic coupled equations is derived.

$$
\dot{\bar{x}}_{i, l}(t)=\left(1 /\left\langle L_{l}(\xi)^{2}\right\rangle\right)\left\langle f_{i}\left(\sum_{j=0}^{P} \bar{x}_{i, j}(t) L_{j}(\xi), \ldots, \sum_{j=0}^{P} \bar{x}_{n, j}(t) L_{j}(\xi), \mu(\xi)\right), L_{l}(\xi)\right\rangle, \begin{gathered}
i=1, \ldots, n \\
l=0, \ldots, P
\end{gathered}
$$

3. Resolve the deterministic system using an appropriate algorithm. 


\subsubsection{Non-intrusive approach}

\section{a. Non-intrusive spectral projection (NISP)}

The NISP method uses the internal product of $x_{i}(t, \xi)$ with $L_{j}(\xi)$ and the orthogonality of Legendre polynomials to compute the stochastic modes $\bar{x}_{i, j}(t)$ from (7) as follows:

$$
\bar{x}_{i, j}(t)=\left(1 /\left\langle L_{j}^{2}(\xi)\right\rangle\right) \int_{-1}^{1} x_{i}(t, \xi) L_{j}(\xi) W(\xi) d \xi
$$

The denominator of expression (11) is an internal product between the same orthogonal polynomial. It can be calculated analytically, then may be tabulated. The problem, however, is related to the calculation of the numerator. Numerical techniques are used for integral computing such as the Monte Carlo or collocation methods (Crestaux et. al, 2009). Note that there are sparse grid collocation techniques used for multidimensional integral computation in cases of multiple uncertain parameters (Ganapathysubramanian and Zabaras, 2007). In the present paper, the studied system (5) possesses one uncertain parameter, so the stochastic modes $\bar{x}_{i, j}(t)$ are computed from mono-dimensional integrals. The Gauss collocation is used for this, (Crestaux et. al, 2009; Jackerman and Roberts, 2009).

\section{b. Regression method}

The regression method consists in calculating the stochastic modes $\bar{x}_{i, j}$ by minimizing the following least square criterion:

$$
\varepsilon=\sum_{k=1}^{\bar{q}}\left[x_{i}\left(t, \xi^{(k)}\right)-\sum_{j=0}^{P} \bar{x}_{i, j}(t) \phi_{j}\left(\xi^{(k)}\right)\right]^{2}, i=1, \ldots, n
$$

with $\bar{q}$ denoting a number of system deterministic simulations such that $P+1<\bar{q}$.

The regression method does not use the orthogonality property of polynomial chaos contrary to the collocation and intrusive methods. It just needs a sample set of the random parameters $\left\{\xi^{(k)}\right\}$ and the corresponding set of simulation outputs $\left\{x_{i}\left(t, \xi^{(k)}\right)\right\}$. The first one can be constructed using Monte 
Carlo techniques while the simulation outputs are obtained following the model describing the physical system (5).

\subsubsection{Statistical characteristics}

Once the stochastic modes have been obtained, they are processed to extract the statistical characteristics of the interest. The first and second order moments are given by:

$$
\left\{\begin{array}{l}
x_{i}^{\text {mean }}(t)=\bar{x}_{i, 0}(t) \\
\sigma_{i}^{2}(t)=\sum_{j=0}^{P}\left(\bar{x}_{i, j}(t)\right)^{2}\left\langle L_{j}^{2}(\xi)\right\rangle-\left(\bar{x}_{i, 0}(t)\right)^{2}, i=1, \ldots, n
\end{array}\right.
$$

As instantaneous histograms describe the probabilistic laws of the system (5), variables can be built from the truncated polynomial chaos expansion (7) using an MC method. No simulation of the system (5) is needed. The procedure simply consists in evaluating the polynomials used in the different samples generated following the distribution law of the random variable. This procedure is clearly less costly than the MC method applied to the system (5).

\subsection{Lyapunov theory}

As mentioned above, this paper deals with the behaviour of the dynamic system described by (5). An important axis in this topic is the stability analysis of the system. The Lyapunov approach to nonlinear systems stability is the most general and most useful tool. It consists in searching for a positive definite function called Lyapunov function, so that its derivative along the system trajectory is negative definite (Slotine, 1999). The main difficulty in this method is to find a suitable Lyapunov function. Sum of square programming has recently entered the literature as a powerful tool to search for polynomial Lyapunov functions for polynomial dynamic systems (Prajna et al, 2005). As the polynomial system (5) is stochastic, the Lyapunov method combined with sum of square (SOS) programming cannot be used directly. An interesting result is given by Fisher who says that the stability of stochastic dynamic systems with polynomial non-linearities can be analyzed by checking for the stability of the deterministic system resulting from the application of intrusive chaos (Fisher 
and Bhattacharya, 2008). The deterministic system resulting from (5) and given by equation (10) is expressed in the following general form:

$$
\dot{\bar{x}}(t)=g(\bar{x}(t))
$$

Where $\bar{x}(t) \in \mathfrak{R}^{n \times(P+1)}$ denotes the vector of the stochastic modes of all the state variables of the system (5). It represents the vector of the state variables of the new deterministic dynamical system (14). $g$ is a vector of polynomial functions in $\bar{x}(t)$ such that $g=\left[\begin{array}{lll}g_{1} & \cdots & g_{n}\end{array}\right]^{T}$. Fisher's statement will be used to analyze the stability of (5). So, the Lyapunov direct method will be summed up, then the sum of square (SOS) polynomials will be described and followed by an illustration about how to use it to construct a polynomial Lyapunov function for a second order cubic system.

\subsubsection{Lyapunov direct method}

Let $D \subset \mathfrak{R}^{n \times(P+1)}$ be a given neighbourhood containing the origin of (14). According to the Lyapunov theorem, if there is a strict positive definite function $V: \mathfrak{R}^{n(P+1)} \rightarrow \mathfrak{R}$ such that:

$$
-\dot{V}(\bar{x})=-(\partial V(\bar{x}) / \partial \bar{x}) g(\bar{x}) \geq 0, \forall \bar{x} \in D
$$

then the origin is a stable equilibrium point.

The main problem is to find a suitable Lyapunov function. Sum of square polynomials can be used to do so.

\subsubsection{Sum of square polynomial}

A multivariate polynomial $p(\bar{x})$ of even degree is said to be a sum of squares (SOS), and so positive definite, if there exists a finite number $m$ of polynomials $p_{1}(\bar{x}), \ldots, p_{m}(\bar{x})$ such that:

$$
p(\bar{x})=\sum_{i=1}^{m} p_{i}^{2}(\bar{x})
$$


The existence of such decomposition is shown to be equivalent to the existence of a real positive semidefinite matrix $Q$ such that :

$$
p(\bar{x})=Z^{T}(\bar{x}) Q Z(\bar{x})
$$

where $Z(\bar{x})$ is the vector of monomials of a degree less than or equal to $\bar{d}=(\operatorname{deg} \operatorname{ree}(p(\bar{x}))) / 2$ :

$$
Z(x)=\left[\begin{array}{c}
\bar{x}_{1}^{\alpha_{1}} \bar{x}_{2}^{\alpha_{2}} \ldots \bar{x}_{n}^{\alpha_{n}} \\
\bar{x}_{1}^{\beta_{1}} \bar{x}_{2}^{\beta_{2}} \ldots \bar{x}_{n}^{\beta_{n}} \\
\vdots
\end{array}\right]
$$

where $\alpha_{i}$ and $\beta_{i}$ are positive numbers such that $\sum_{i=1}^{n} \alpha_{i} \leq \bar{d}$ and $\sum_{i=1}^{n} \beta_{i} \leq \bar{d}$.

As a consequence of the above definition, SOS decomposition is reduced to the calculation of matrix $Q$ with an SOS program which can be solved using SOSTOOLS specifically developed software (Prajna et. al, 2005).

\subsubsection{Construction of Lyapunov function with SOS decomposition}

SOS programming has been used to search for polynomial Lyapunov functions in polynomial dynamic systems (Prajna et al, 2005). His main idea is to replace checking for positivity conditions on $V(x)$ and $-\dot{V}(x)$ of the Lyapunov theorem by equivalent SOS conditions. Then, for the polynomial system (14), the origin is stable if there exists a polynomial function $V: \mathfrak{R}^{n(P+1)} \rightarrow \mathfrak{R}$ such that it satisfies the following two conditions:

$$
\begin{aligned}
& V(\bar{x})-\varphi(\bar{x}) \text { is SOS } \\
& -\dot{V}(x)=-(\partial V(\bar{x}) / \partial \bar{x}) g(\bar{x}) \text { is SOS }
\end{aligned}
$$

where $\varphi(\bar{x})$ is a strict positive polynomial function used to ensure the strict positivity of $V(\bar{x})$. Let $2 \bar{d}$ be the degree of $V(\bar{x})$. A simple choice of $\varphi(\bar{x})$ is given by (18). 


$$
\varphi(\bar{x})=\sum_{i=1}^{n} \sum_{j=1}^{\bar{d}} \varepsilon_{i j} \bar{x}_{i}^{2 j}
$$

where $\varepsilon_{i j} \geq 0$ are constants for all $i$ and $j$, (Prajna et. al, 2005).

\section{General example}

In order to understand how the SOS programming based method is used to search for a polynomial Lyapunov function, consider a second order polynomial dynamic system expressed by the following general form:

$$
\left\{\begin{array}{l}
\dot{x}_{1}=a_{11} \bar{x}_{1}+a_{12} \bar{x}_{2}+b_{11} \bar{x}_{1}^{2}+b_{12} \bar{x}_{1} \bar{x}_{2}+b_{13} \bar{x}_{2}^{2}+c_{11} \bar{x}_{1}^{3}+c_{12} \bar{x}_{1}^{2} \bar{x}_{2}+c_{13} \bar{x}_{1} \bar{x}_{2}^{2}+c_{14} \bar{x}_{2}^{3} \\
\dot{\bar{x}}_{2}=a_{21} \bar{x}_{1}+a_{22} \bar{x}_{2}+b_{21} \bar{x}_{1}^{2}+b_{22} \bar{x}_{1} \bar{x}_{2}+b_{23} \bar{x}_{2}^{2}+c_{21} \bar{x}_{1}^{3}+c_{22} \bar{x}_{1}^{2} \bar{x}_{2}+c_{23} \bar{x}_{1} \bar{x}_{2}^{2}+c_{24} \bar{x}_{2}^{3}
\end{array}\right.
$$

For simplicity's sake, a quadratic Lyapunov function is searched for, with no constant and no linear terms. This ensures the positivity of the function searched. Such a Lyapunov function can be expressed by:

$$
V(\bar{x})=d_{20} \bar{x}_{1}^{2}+d_{11} \bar{x}_{1} \bar{x}_{2}+d_{02} \bar{x}_{2}^{2}
$$

Note that in the following the polynomial function $\varphi(x)$ is not used, so as to make the procedure easier to understand.

The Lyapunov function (20) can be written in an SOS representation as given by (17):

$$
V(\bar{x})=\frac{1}{2} Z^{T}(\bar{x}) G Z(\bar{x})
$$

where $Z(\bar{x})$ is the vector of monomials of a degree less than 2 (degree of $V(\bar{x})$ ); consequently, $Z(\bar{x})$ is the same as the state vector $Z(\bar{x})=\left[\begin{array}{ll}\bar{x}_{1} & \bar{x}_{2}\end{array}\right]^{T}$, and $G=\left[\begin{array}{cc}2 d_{20} & d_{11} \\ d_{11} & 2 d_{02}\end{array}\right]$. The condition for the existence of a SOS representation for $V(\bar{x})$ is that $G \geq 0$. 
The Lyapunov function derivative can be found as follows:

$$
\begin{aligned}
\dot{V}(\bar{x})= & t_{20} \bar{x}_{1}^{2}+t_{11} \bar{x}_{1} \bar{x}_{2}+t_{02} \bar{x}_{2}^{2}+t_{30} \bar{x}_{1}^{3}+t_{21} \bar{x}_{1}^{2} \bar{x}_{2}+t_{12} \bar{x}_{1} \bar{x}_{2}^{2}+t_{03} \bar{x}_{2}^{3}+t_{40} \bar{x}_{1}^{4}+t_{31} \bar{x}_{1}^{3} \bar{x}_{2}+t_{22} \bar{x}_{1}^{2} \bar{x}_{2}^{2}+ \\
& t_{13} \bar{x}_{1} \bar{x}_{2}^{3}+t_{04} \bar{x}_{2}^{4}
\end{aligned}
$$

where $t_{i j}$ are linear functions of the system parameters $\left\{a_{i j}, b_{i j}, c_{i j}\right\}$ and the Lyapunov function coefficients $\left\{d_{20}, d_{11}, d_{02}\right\}$. They are obtained as follows:

$$
\begin{array}{ll}
t_{11}=d_{11} a_{11}+d_{11} a_{22}+2 d_{20} a_{12}+2 d_{02} a_{21}, & t_{12}=2 d_{20} b_{13}+d_{11} b_{12}+d_{11} b_{23}+2 d_{02} b_{22}, \\
t_{13}=2 d_{20} c_{14}+d_{11} c_{13}+d_{11} c_{24}+2 d_{02} c_{23}, & t_{04}=2 d_{02} c_{24}+d_{11} c_{14}, \\
t_{03}=2 d_{02} b_{23}+d_{11} b_{13}, & t_{20}=2 d_{20} a_{11}+d_{11} a_{21}, \\
t_{30}=2 d_{20} b_{11}+d_{11} b_{21}, & t_{40}=2 d_{20} c_{11}+d_{11} c_{21}, \\
t_{02}=2 d_{02} a_{22}+d_{11} a_{12}, & t_{21}=2 d_{20} b_{12}+d_{11} b_{11}+d_{11} b_{22}+2 d_{02} b_{21}, \\
t_{22}=2 d_{20} c_{13}+d_{11} c_{12}+d_{11} c_{23}+2 d_{02} c_{22}, & t_{31}=2 d_{20} c_{12}+d_{11} c_{11}+d_{11} c_{22}+2 d_{02} c_{21} .
\end{array}
$$

An SOS representation of the Lyapunov function derivative can then be considered as follows:

$$
\dot{V}(\bar{x})=-(1 / 2) H(\bar{x}) R H(\bar{x})
$$

where $H(\bar{x})=\left[\begin{array}{lllll}\bar{x}_{1} & \bar{x}_{2} & \bar{x}_{1}^{2} & \bar{x}_{1} \bar{x}_{2} & \bar{x}_{2}^{2}\end{array}\right]^{T}$ and matrix $R$ is obtained as follows:

$$
R=\left[\begin{array}{ccccc}
2 t_{20} & t_{30} & t_{21}+v_{1} & t_{12}+v_{2} & t_{11} \\
t_{30} & 2 t_{40} & t_{31} & -v_{3} & -v_{1} \\
t_{21}+v_{1} & t_{31} & 2 t_{22}+2 v_{3} & t_{13} & -v_{2} \\
t_{12}+v_{2} & -v_{3} & t_{13} & 2 t_{04} & t_{03} \\
t_{11} & -v_{1} & -v_{2} & t_{03} & 2 t_{02}
\end{array}\right]
$$

where $v_{i(i=1,2,3)}$ are arbitrary real parameters. See (Prajna et. al, 2005) for further discussion about how to introduce these parameters. 
It must be noted that the constant term in the monomial vector $H(\bar{x})$ is omitted as there is no constant and no linear term in the derivative obtained.

By replacing $t_{i j}$ with the linear functions obtained above, matrix $R$ will be dependent on the system parameters, the Lyapunov function coefficients and the $v_{i}$ arbitrary parameters.

According to the Lyapunov theorem, the origin will be stable if $-\dot{V}(\bar{x})$ is positive semi definite. The SOS condition $R \geq 0$ must then be fulfilled.

The problem is then to search for the Lyapunov function coefficients $d_{02}, d_{11}, d_{02}$ and the arbitrary $v_{i}$ parameters, such that the matrices $G$ and $R$ will be positive and semi positive definite respectively. The resolution process represents the feasibility problem of an SOS program which is implemented and solved, using the Sostools toolbox under Matlab, (Prajna et. al, 2005). Note that for polynomial dynamic systems of a higher order, the same problem with more unknowns must be solved.

\section{Application}

In order to check the capabilities of the polynomial chaos approach in the analysis of dynamic behaviours of stochastic nonlinear friction systems, a simple self-excited mechanism (Fig.1) proposed by Hultén and Sinou will be used to study squeal vibrations in drum brakes (Hultén, 1993; Sinou and Jezequel, 2007). The aim is to use the polynomial chaos approach in two complementary problems: the stability analysis and limit cycle dispersion analysis. The two problems are considered according to uniform dispersions of the friction coefficient within two uncertain intervals: stable and unstable intervals. The polynomial chaos is used to analyze the stability of the system in the first interval and to estimate the amplitudes of oscillations in the second interval in which the system presents flutter instability.

Location of Fig.1. 


\subsection{Description of the mechanical model}

Hultèn's model is composed of a mass $m$ held against a moving band; the contact between the mass and the band is modelled by two plates supported by two different springs. For simplicity's sake, it is assumed that the mass and band surfaces always keep in contact. This assumption is justified by a preload applied to the system. The contact can be expressed by two cubic stiffnesses. Damping is integrated as shown in Fig.1. The friction coefficient at contact is assumed to be constant and the band moves at a constant velocity. Then it is assumed that the direction of the friction force does not change because the relative velocity between the band speed and $\dot{X}_{1}$ or $\dot{X}_{2}$ is assumed to be positive.

The friction coefficient is assumed to be constant but uncertain. This uncertainty is related in general to the manufacturing process which yields uncertain tribological characteristics for the contact surfaces. So for two manufactured systems, the corresponding friction coefficients are constant but with different values since the tribological characteristics are different.

The tangential force $F_{T}$ due to friction contact is assumed to be proportional to the normal force $F_{N}$ as given by Coulomb's law: $F_{T}=\mu F_{N}$. Assuming that the normal $F_{N}$ is linearly related to the displacement of the mass normal to the contact surface, the resulting equations of motion can be expressed as: (Sinou and Jezequel, 2007).

$$
\left[\begin{array}{ll}
1 & 0 \\
0 & 1
\end{array}\right]\left(\begin{array}{l}
\ddot{X}_{1} \\
\ddot{X}_{2}
\end{array}\right)+\left[\begin{array}{cc}
\eta_{1} \omega_{1} & 0 \\
0 & \eta_{2} \omega_{2}
\end{array}\right]\left(\begin{array}{l}
\dot{X}_{1} \\
\dot{X}_{2}
\end{array}\right)+\left[\begin{array}{cc}
\omega_{1}^{2} & -\mu \omega_{2}^{2} \\
\mu \omega_{1}^{2} & \omega_{2}^{2}
\end{array}\right]\left(\begin{array}{l}
X_{1} \\
X_{2}
\end{array}\right)=\left(\begin{array}{l}
-\varphi_{1}^{N L} X_{1}^{3}+\mu \varphi_{2}^{N L} X_{2}^{3} \\
-\mu \varphi_{1}^{N L} X_{1}^{3}-\varphi_{2}^{N L} X_{2}^{3}
\end{array}\right)
$$

where $\eta_{i}=c_{i} / \sqrt{m k_{i}}$ are the relative damping coefficients, $\omega_{i}=\sqrt{k_{i} / m}$ are the natural pulsations and $\varphi_{i}^{N L}=k_{i}^{N L} / m$ for $i=1,2$

For numerical application: $\omega_{1}=2 \pi \times 100 \mathrm{rad} / \mathrm{s}, \omega_{2}=2 \pi \times 75 \mathrm{rad} / \mathrm{s}, \eta_{1}=\eta_{2}=0.02, \varphi_{1}^{N L}=\omega_{1}^{2}$ and $\varphi_{2}^{N L}=0$

Considering $X_{1}=x_{1}, \dot{X}_{1}=x_{2}, X_{2}=x_{3}$ and $\dot{X}_{2}=x_{4}$, the system model is expressed with a state space representation as:

$$
\dot{x}(t)=A(\mu) x(t)+f_{N L}(x(t), \mu)
$$


where $x(t)=\left[\begin{array}{llll}x_{1}(t) & x_{2}(t) & x_{3}(t) & x_{4}(t)\end{array}\right]^{T}, A(\mu)=\left[\begin{array}{cccc}0 & 1 & 0 & 0 \\ -\omega_{1}^{2} & -\eta_{1} \omega_{1} & \mu \omega_{2}^{2} & 0 \\ 0 & 0 & 0 & 1 \\ -\mu \omega_{1}^{2} & 0 & -\omega_{2}^{2} & -\eta_{2} \omega_{2}\end{array}\right]$ and $f_{N L}(x(t), \mu)=\left[\begin{array}{c}0 \\ -\varphi_{1}^{N L} x_{1}^{3}(t)+\mu \varphi_{2}^{N L} x_{3}^{3}(t) \\ 0 \\ -\mu \varphi_{1}^{N L} x_{1}^{3}(t)-\varphi_{2}^{N L} x_{3}^{3}(t)\end{array}\right]$

\subsection{Stability analysis}

For a designer, the main objective of a robust stability analysis is to define with certainty that the system studied is stable for an uncertain parameter defined within an uncertain interval (such as the dispersion interval of the friction coefficient). Therefore, the classic deterministic approach is often used. This parametric study consists in calculating the eigenvalues of the linearized system at each value of the uncertain parameter, then stability is analyzed by testing the sign of the real parts of the eigenvalues obtained. This procedure becomes difficult and prohibitive for nonlinear systems of a higher order, since the calculation of the corresponding eigenvalues goes through the resolution of characteristic equations of higher order and numerous samples of the uncertain parameter are necessary. Two main limits characterize the deterministic approach. The first one is that in practice there is no possibility to test all possible values of an uncertain parameter; the second one is that the method is not conclusive about stability when eigenvalues are imaginary. So, the parametric approach gives no certainty on the system stability.

The principal objective of using intrusive chaos combined with the direct Lyapunov approach is to define the intervals of stability with certainty. This method is proposed by Fisher. (2008) and has been tested on numerical examples only. The main idea is that the stability of a stochastic dynamic 
system with polynomial non-linearities can be analyzed through the stability analysis of the deterministic dynamic system resulting from applying intrusive polynomial chaos.

\subsubsection{Parametric study of stability}

The stability of the friction system equilibrium is investigated using a classic parametric method. The friction coefficient is assumed to have dispersions within the interval [ $\left[\begin{array}{ll}0 & 0.6\end{array}\right]$.

The equilibrium $x_{e}$ of the system (26) is obtained by solving the non-linear static equation:

$$
\dot{x}_{e}=A(\mu) x_{e}+f_{N L}\left(x_{e}, \mu\right)=0 .
$$

The non-linear static solution corresponds to the origin of the system (26). So, the eigenvalues $\lambda$ of the linearized system can be found by solving the characteristic equation:

$$
\operatorname{det}(A(\mu)-\lambda I)=0
$$

The evolution of the system eigenvalues is plotted against the values of the friction coefficient in Fig.2.

Location of Fig.2.

As long as the real parts of all the eigenvalues remain negative, the static solution of the system is stable. When at least one of the eigenvalues has a positive real part, the static solution is unstable. The imaginary parts of these eigenvalues represent instability frequencies.

The later study of the stability consisted in creating a vector of values from the interval of the uncertain friction coefficient $\mu$. The conditions for the stability have been tested for each point in the vector. For the tested values of $\mu$ which belong to $[0,0.28]$, the origin is stable while for the values greater than 0.28 , the origin is unstable. This conclusion is true for the created vector but, in fact, cannot be extended to the whole interval $[0,0.6]$ in which there exist values for which stability have not been tested since they do not belong to the generated vector. So no certainty can be obtained for the stability conclusions in the considered interval. 
The size of the generated vector can be increased to have more certainty on the stability analysis. However, this yields in practice considerable computational difficulties in particular when dealing with more complex dynamic systems.

\subsubsection{Robust approach}

In order to obtain a certain interval of stability with Fisher's method, stability analysis is considered not on a single interval but on several intervals. This is to reduce computational difficulties. In fact, as the order of the Wiener-Legendre expansion required to well approximate the statistics of the system (26) augments with the increase of the uncertain interval bandwidth, the dimension of the deterministic system resulting from the application of the intrusive chaos becomes important. Consequently, the number of monomials in the polynomial Lyapunov function to be searched for is so large that the necessary memory size of storage must be higher. So, three intervals $\left[\begin{array}{ll}a & b\end{array}\right]$ are considered:[ $\left[\begin{array}{ll}0 & 0.1\end{array}\right],\left[\begin{array}{ll}0.1 & 0.2\end{array}\right]$ and $\left[\begin{array}{ll}0.2 & 0.28\end{array}\right]$. For each interval, a uniform dispersion of the friction coefficient is assumed which helps to express it as:

$$
\mu(\xi)=\mu_{\text {moy }}+\alpha \xi
$$

where $\mu_{m o y}=(a+b) / 2, \alpha$ is a suitable constant and $\xi \in\left[\begin{array}{ll}-1 & 1\end{array}\right]$ is a uniform stochastic variable. Once the Wiener-Legendre expansion is applied, a deterministic system of dynamic equations is obtained following the procedure described in Section 2.2. For each interval, the order of the development is validated by comparing the mean values and the variances of the system variables (displacement and velocity) obtained from the Wiener-Legendre expansion to MC solutions. SOS programming is then used to build the polynomial Lyapunov functions proving the stability of the system origin.

The deterministic system resulting from the application of the Wiener-Legendre expansion is given by: 


$$
\left\{\begin{array}{c}
\dot{\bar{x}}_{1, l}=\bar{x}_{2, l} \\
\dot{\bar{x}}_{3, l}=\bar{x}_{4, l} \\
\dot{\bar{x}}_{2, l}=-\eta_{1} \omega_{1} \bar{x}_{2, l}-\omega_{1}^{2} \bar{x}_{1, l}+\left(1 /\left\langle L_{l}^{2}\right\rangle\right)\left(\omega_{2}^{2} \sum_{i=0}^{P} \bar{x}_{3, j}\left\langle\mu L_{j} L_{l}\right\rangle-\varphi_{1}^{N L} \sum_{j=0}^{P} \sum_{k=0}^{P} \sum_{q=0}^{P} \bar{x}_{1, j} \bar{x}_{1, k} \bar{x}_{1, q}\left\langle L_{j} L_{k} L_{q} L_{l}\right\rangle\right) \\
-\left(\varphi_{2}^{N L} /\left\langle L_{l}^{2}\right\rangle\right) \sum_{j=0}^{P} \sum_{k=0}^{P} \sum_{q=0}^{P} \bar{x}_{3, j} \bar{x}_{3, k} \bar{x}_{3, q}\left\langle\mu L_{j} L_{k} L_{q} L_{l}\right\rangle \\
\dot{\bar{x}}_{4, l}=-\eta_{2} \omega_{2} \bar{x}_{4, l}-\omega_{2}^{2} \bar{x}_{3, l}+\left(1 /\left\langle L_{l}^{2}\right\rangle\right)\left(\omega_{1}^{2} \sum_{i=0}^{P} \bar{x}_{1, j}\left\langle\mu L_{j} L_{l}\right\rangle-\varphi_{1}^{N L} \sum_{j=0}^{P} \sum_{k=0}^{P} \sum_{q=0}^{P} \bar{x}_{1, j} \bar{x}_{1, k} \bar{x}_{1, q}\left\langle\mu L_{j} L_{k} L_{q} L_{l}\right\rangle\right) \\
-\left(\varphi_{2}^{N L} /\left\langle L_{l}^{2}\right\rangle\right) \sum_{j=0}^{P} \sum_{k=0}^{P} \sum_{q=0}^{P} \bar{x}_{3, j} \bar{x}_{3, k} \bar{x}_{3, q}\left\langle L_{j} L_{k} L_{q} L_{l}\right\rangle \\
l=0,1, \ldots, P
\end{array}\right.
$$

The results corresponding to the interval $\left[\begin{array}{ll}a & b\end{array}\right]=\left[\begin{array}{ll}0 & 0.1\end{array}\right]$ are shown below.

For $P=3$ mean values $\hat{\mu}_{X_{1}}(t), \hat{\mu}_{\dot{X}_{1}}(t)$ and variances $\sigma_{X_{1}}{ }^{2}(t), \sigma_{\dot{X}_{1}}^{2}(t)$ respectively of the displacement $X_{1}(t)$ and the velocity $\dot{X}_{1}(t)$ are computed after resolving the differential system (30). The latter operation is performed using Matlab's ODE45 solver. The computed mean values and variances are represented in Fig.3 and Fig.4 respectively as functions of time $t \in\left[\begin{array}{ll}0 & t_{\max }\end{array}\right]$ with $t_{\max }=3 s$. Moreover, they are compared with a MC solution obtained from simulations with $N=10,000$ uniformly distributed samples within the given interval.

\section{Location of Fig.3}

Location of Fig.4

As shown in Fig. 3 and Fig.4, the mean values $\hat{\mu}_{X_{1}}(t), \hat{\mu}_{\dot{X}_{1}}(t)$ and variances $\sigma_{X_{1}}{ }^{2}(t), \sigma_{\dot{X}_{1}}^{2}(t)$ obtained from the third order Wiener-Legendre expansion, are as accurate as the referential solutions obtained by the MC method. The two statistic moments show oscillatory transients until they damp to steady state. Asymptotic decay of the mean values and the variances is well approximated by the third order Wiener-Legendre expansion. The deterministic system (30) is used to analyze the stability of the stochastic dynamic system (26). SOS programming and Sostools help to obtain a quadratic Lyapunov function is given by: 


$$
\begin{aligned}
V(\bar{x})= & 0.38 \bar{x}_{10}^{2}+1.39 \bar{x}_{30}^{2}+1.37 \bar{x}_{31}^{2}+1.38 \bar{x}_{32}^{2}+1.34 \bar{x}_{33}^{2}-0.78 e^{-1} \bar{x}_{10} \bar{x}_{12}-0.68 e^{-1} \bar{x}_{11} \bar{x}_{13} \\
& -0.10 e^{-1} \bar{x}_{13} \bar{x}_{32}-0.18 e^{-1} \bar{x}_{31} \bar{x}_{32}-0.44 e^{-1} \bar{x}_{12} \bar{x}_{33}-0.29 e^{-1} \bar{x}_{13} \bar{x}_{33}-0.38 e^{-1} \bar{x}_{10} \bar{x}_{31} \\
& -0.34 e^{-1} \bar{x}_{11} \bar{x}_{31}-0.22 e^{-1} \bar{x}_{30} \bar{x}_{31}-0.20 e^{-1} \bar{x}_{11} \bar{x}_{32}-0.29 e^{-1} \bar{x}_{12} \bar{x}_{32}-0.43 e^{-1} \bar{x}_{10} \bar{x}_{30} \\
& -0.11 e^{-1} \bar{x}_{11} \bar{x}_{30}-0.16 e^{-1} \bar{x}_{32} \bar{x}_{33}+0.23 \bar{x}_{13}^{2}+0.28 \bar{x}_{11}^{2}+0.22 \bar{x}_{12}^{2}
\end{aligned}
$$

This proves the stability of the origin for a friction coefficient within $\left[\begin{array}{ll}0 & 0.1\end{array}\right]$. The same conclusion is obtained for the two other intervals. The results are given in Annex. So, stability can be established for the considered dispersion interval of the friction coefficient $\left[\begin{array}{ll}0 & 0.28\end{array}\right]$.

A more general conclusion can be drawn on the complementarity of both the parametric study and the intrusive chaos based approach. As mentioned previously, the first method presents some limits which do not provide full certainty on stability. The second method cannot be conclusive either on the stability if there are instability points within the uncertain interval of the control parameter. So, in practice both methods can be combined for greater efficiency and more certainty. Indeed, the parametric study can be carried out with a small number of samples in a given dispersion interval to determine an interval in which no instability points appear, then the intrusive chaos based method can be used to confirm stability within the totality of the given interval.

\subsection{Limit cycle analysis}

For friction systems, it is not always sufficient to obtain stable and instable zones is not always sufficient (Sinou, 2006, paragraph 5). Indeed, for a good design, it must be possible to determine the amplitudes of limit cycle oscillations (LCO) to know if they can be neglected or not. The main objective of this study is to quantify the resulting dispersion of the LCOs.

For an uncertain friction coefficient (defined by a uniform law on a given interval), it is necessary to estimate the mean value, the standard deviation, the minimum and the maximum values of displacements and velocities with the intrusive and the non-intrusive approaches. 
Consider an interval $\left[\begin{array}{ll}a & b\end{array}\right]$ for $\mu$ in which the system (26) presents flutter instability. For example $\left[\begin{array}{ll}a & b\end{array}\right]=\left[\begin{array}{ll}0.3 & 0.33\end{array}\right]$. First, the sensitivity of LCO amplitudes to small variations of the friction coefficient is investigated. The solutions of the system (26) corresponding to $\mu=0.3$ and $\mu=0.31$ are computed using the ODE45 solver. The corresponding instantaneous displacements $X_{1}(t)$ and the limit cycles $\left(X_{1}, \dot{X}_{1}\right)$ are plotted in Fig.5 and Fig.6 respectively. As the stationary behaviour (LCO) is reached after a half second (Fig. 5), simulation of the system (26) is stopped at 3 sec.

Location of Fig.5

Location of Fig.6

In Fig.5 and Fig.6, a variation of 3.3\% of the friction coefficient involves a variation of almost $40 \%$ in the amplitude of the LCOs $\left(X_{1}, \dot{X}_{1}\right)$.

\subsubsection{Study with intrusive approach}

Statistics in terms of mean value and standard deviation of LCO are estimated using (15) after solving the system (30) using ODE45. As the stationary behaviour (LCOs) is reached after a half second (Fig. 5), simulation of (30) is also reached after three seconds. All These operations are carried out for $P=10, P=15$ and $P=20$ to observe the influence of the order of the Wiener-Legendre expansion used. The mean values of responses $X_{1}(t)$ and $\dot{X}_{1}(t)$ obtained with the different orders are shown in Fig.7 as functions of time $t$ and they are compared with the referential solutions obtained with the MC method by using 0,000 uniformly distributed samples within [ $\left.\begin{array}{ll}0.3 & 0.33\end{array}\right]$.

\section{Location of Fig.7}

The uncertainty of the friction coefficient affects the amplitude and the frequency of the system responses (displacements and velocities). The effect on the frequency is observed from the variations 
of the phase difference between the system responses corresponding to the samples of the friction coefficient. These responses are functions of time and are of opposite signs so that they cancel each other when the mean value is computed with the MC method. Consequently, the mean value is a damped oscillation as shown in Fig.7. It can be noted that this damping and the amplitudes of the mean values are approximated more accurately with the $20^{\text {th }}$ Wiener-Legendre expansion than the $10^{\text {th }}$ and $15^{\text {th }}$ Wiener-Legendre expansions. The accuracy of the Wiener-Legendre expansion decreases with time and thus requires a higher order. The same observation applies to Fig. 8 where the probabilistic distribution of $X_{1}(t)$ is reconstructed at different times $t=1 \mathrm{sec}, t=2 \mathrm{sec}$ and at $t=3 \mathrm{sec}$. Note that no simulation of the system is needed to construct the probabilistic distribution. It just needs the evaluation of the Legendre polynomial at the given samples of the stochastic variable used.

Location of Fig.8

The $95 \%$ confidence interval estimated for the mean value of $X_{1}(t)$ obtained from the MC method is represented in Fig.9.

Location of Fig.9

The referential mean value is always included in the constructed confidence interval contrary to the mean value obtained by the intrusive PC with $P=20$. So, it appears that the PC solution for the mean value with $P=20$ is not suitable.

\subsubsection{Non-intrusive approach}

To improve the accuracy of the intrusive Wiener-Legendre approximation, the expansion order can be increased. The large size of the resulting deterministic system makes this operation difficult. Indeed, the intrusive application of the Wiener-Legendre expansion with an order greater than 20 gives a deterministic system with more than 80 dynamic equations, so implementation and resolving become 
difficult tasks. The non-intrusive approach can be seen as an alternative to circumvent these drawbacks since no modifications of the system model are needed. The NISP and regression methods with $P=20$ are applied. The number of simulations required for the first method is given by $P+1$ and corresponds to the zeros of the $21^{\text {st }}$ order Legendre polynomial. For the regression method, the number is fixed arbitrarily to 25 and corresponds to the zeros of the $25^{\text {th }}$ order Legendre polynomial. Both numbers are much smaller than the one necessary for an MC method with good accuracy.

The mean values obtained with all the methods are shown in Fig.10 as functions of time $t$ and the distributions of $X_{1}(t)$ at $t=1 s, t=2 s$ and $t=3 s$ are constructed and represented in Fig.11.

\section{Location Fig.10}

Location of Fig.11

In Fig.10, the mean values of $X_{1}(t)$ computed from non-intrusive methods (collocation and regression) are almost similar to that of the MC method for all times $t \in[0,3 \mathrm{sec}]$, contrary to the intrusive solution for which the relative error rises to a higher level (from $16.94 \%$ at $t=1 \mathrm{~s}$ to more than $100 \%$ at $t=3 s)$. The probability distributions of $X_{1}(t)$ at different times obtained with non intrusive methods are the best approximations, as illustrated in Fig.11. The regression method gives the most accurate results with smaller maximal relative errors $(7.14 \%$ for the mean value and $0.11 \%$ for the standard deviation. From Fig.12, the mean values obtained with both the collocation and regression methods belong to the $95 \%$ confidence interval. This confirms the improvement of the accuracy given by the non-intrusive methods.

\section{Location of Fig.12}

In a previous step, polynomial chaos was used intrusively and non-intrusively to estimate the first and second order statistics of the dynamic behaviour of the friction system (26). The second manner was shown to be more efficient and more accurate than the first one. The second objective of the study 
is to estimate the minimum and maximum of the LCO amplitudes. Therefore, the $20^{\text {th }}$ and the $30^{\text {th }}$ Wiener-Legendre expansions are used to construct the minimum and maximum distributions of the LCO amplitudes with 10,000 samples distributed uniformly within the orthogonality interval of the Legendre polynomial. The global minimum and maximum obtained with $P=20$ and $P=30$ are given in Tab.1 and Tab.2, respectively. Note that this method is less costly than the MC procedure. The latter requires the system's simulation for each sample of the uncertain parameter contrary to the polynomial chaos based approach which requires only the evaluation of the Legendre polynomials at the generating samples.

\section{Location of Tab.1}

Location of Tab.2

For $P=20$, all methods suitably estimate the reference values of the global minimum and maximum obtained with the Monte Carlo method. Non intrusive methods give estimations with smaller relative errors than the ones obtained by the intrusive method.

For $P=30$, only non intrusive methods are used since the intrusive method is, as shown previously, less accurate. Moreover, the latter is more difficult to implement. The results given by the nonintrusive methods show great accuracy which consists of small relative error $(<0.6 \%)$.

\section{Conclusion:}

An approach based on the polynomial chaos theory has been proposed to study the dynamic behaviour of friction systems which are highly sensitive to dispersions of the friction laws. A complete study of the dynamic behaviour including stability and vibratory analyses has been carried out for a two degree of freedom model describing a drum brake system characterized by an uncertain friction coefficient. The intrusive and non-intrusive methods based on the Wiener-Legendre expansion have been used to determine stability intervals and to predict the amplitudes of limit cycle oscillations in a flutter instability zone. Both problems have been dealt with according to an uncertain friction coefficient. The efficiency of the proposed methods compared with the prohibitive Monte Carlo 
method has been highlighted. The main results of the present study show that the polynomial chaos may be an efficient tool to take into account the dispersions of the friction coefficient in the dynamic behaviour study of friction systems. First, the direct Lyapunov method combined with the intrusive Wiener-Legendre expansion can be used efficiently to analyze stability according to the dispersion of the friction coefficient. In fact, this method helps to overcome the limits of the parametric approach based on the sample per sample stability analysis. The first step is to apply the polynomial chaos intrusively to obtain a deterministic system of dynamic equations corresponding to an uncertain interval; then the second step is to use the direct Lyapunov method in particular SOS decompositions to find a Lyapunov function for the system obtained. The second problem dealt with in this paper is the estimation of the extreme of LCO amplitudes according to a given uncertain interval of the friction coefficient in which the system presents flutter instability. In this field, the polynomial chaos approach in particular, the non-intrusive methods have also given satisfaction in terms of accuracy of the estimations. Finally, it is important to note that the limit cycles are reached rapidly, so the LCO analysis is related to the global problem which consists in the analysis of short term statistics in stochastic dynamic systems. An interesting perspective is to investigate the case were LCOs are reached after a longer time. Further work in this context is in progress.

\section{Annex}

In the stability study presented in Subsection 3.2.2, the quadratic Lyapunov functions found for the intervals $\left[\begin{array}{ll}0.1 & 0.2\end{array}\right]$ and $\left[\begin{array}{ll}0.2 & 0.28\end{array}\right]$ are obtained respectively as:

$$
\begin{aligned}
V_{2}(\bar{x}) & =0.27 \bar{x}_{11}^{2}+0.22 \bar{x}_{13}^{2}-0.06 \bar{x}_{11} \bar{x}_{13}+0.22 \bar{x}_{12}^{2}+1.24 \bar{x}_{30}^{2}+1.23 \bar{x}_{31}^{2}+1.23 \bar{x}_{32}^{2}+1.21 \bar{x}_{33}^{2}-0.11 \bar{x}_{10} \bar{x}_{30} \\
& +0.01 \bar{x}_{12} \bar{x}_{30}-0.02 \bar{x}_{10} \bar{x}_{31}-0.08 \bar{x}_{11} \bar{x}_{31}+0.01 \bar{x}_{13} \bar{x}_{31}-0.05 \bar{x}_{30} \bar{x}_{31}+0.01 \bar{x}_{10} \bar{x}_{32}-0.07 \bar{x}_{12} \bar{x}_{32} \\
& -0.04 \bar{x}_{31} \bar{x}_{32}-0.03 \bar{x}_{12} \bar{x}_{33}-0.07 \bar{x}_{13} \bar{x}_{33}-0.03 \bar{x}_{32} \bar{x}_{33}+1.23 \bar{x}_{33}^{2}
\end{aligned}
$$




$$
\begin{aligned}
V_{3}(\bar{x})= & 1.07 \bar{x}_{31}^{2}+1.05 \bar{x}_{33}^{2}-0.04 \bar{x}_{31} \bar{x}_{30}-0.03 \bar{x}_{33} \bar{x}_{32}-0.07 \bar{x}_{10} \bar{x}_{12}+1.08 \bar{x}_{30}^{2}+0.27 \bar{x}_{11}^{2}+0.01 \bar{x}_{30} \bar{x}_{12} \\
& -0.14 \bar{x}_{30} \bar{x}_{10-}-0.06 \bar{x}_{11} \bar{x}_{13}-0.09 \bar{x}_{32} \bar{x}_{12}+0.01 \bar{x}_{32} \bar{x}_{10}+0.22 \bar{x}_{13}^{2}-0.01 \bar{x}_{31} \bar{x}_{10}-0.11 \bar{x}_{31} \bar{x}_{11}+ \\
& -0.01 \bar{x}_{31} \bar{x}_{13}+1.07 x_{32}^{2}+0.22 \bar{x}_{12}^{2}+0.37 \bar{x}_{10}^{2}-0.004 \bar{x}_{32} \bar{x}_{31}+-0.02 \bar{x}_{33} \bar{x}_{12}+0.01 \bar{x}_{33} \bar{x}_{11}-0.09 \bar{x}_{33} \bar{x}_{13}
\end{aligned}
$$

Sébastien Berger gratefully acknowledges the financial support of the French National

Research Agency through the Young Researcher program ANR-07-JCJC-0059-01-CSD2.

\section{Reference}

Askey, R., Wilson, J., 1985. Some Basic Hypergeometric Polynomials that Generalize Jacobi Polynomials. Memoirs Amer. Math. Society. Soc. 319.

Babuska, I., Tempone, R., Zouraris, G.E., 2004. Galerkin finite element approximation of stochastic elliptic partial differential equations. SIAM J. Scientific Comput. 24, 619-644.

Babuska, I., Nobile, F., Tempone, R., 2007. A stochastic collocation method for elliptic partial differential equations with random input data. SIAM J. Num. Ana. 45, 1005-1034.

Beran, P. S., Pettit, C. L., Millman, D. L., 2006. Uncertainty quantification of limit-cycle oscillations. J. Comput.Phys. 217, 217-247.

Blanchard, E., Sandu, A., Sandu, C., 2009. Parameter estimation for mechanical systems via an explicit representation of uncertainty. Int. J. for Computer-Aided Engineering Computation. 26, 541569.

Blanchard, E., Sandu, A., Sandu, C., 2010. Polynomial chaos-based parameter estimation methods applied to vehicle system. Proc. Of the Institution of Mechanical Engineers Part K: J. of Multi-Body Dynamics. 224, 59-81.

Blanchard, E., Sandu, A., Sandu, C., 2010. A polynomial chaos-based Kalman filter approach for parameter estimation of mechanical systems. ASME J. Dyn. Sys. Mea. Control, Special Issue on Physical System Modeling. 132, 18 pages.

Cameron, H., Martin, W., 1947. The orthogonal development of nonlinear functionals in series of Fourier-Hermite functional. Ann. Math. 48, 385-392. 
Chevennement-Roux, C., Dreher, T., Alliot, P., Aubry, E., Lainé , J-P., Jézéquel, L., 2007. Flexible wiper system system dynamic instabilities: modeling and experimental validation. Experimental Mechanics. 47, 201-210.

Chevennement-Roux, C., Grenouillat, R., Dreher, T., Alliot, P., Aubry, E., Lainé, J-P., Jézéquel, L., 2005. Wiper system with flexible structures-Instabilities analysis and correlation with a theoretical model. SAE transactions. 114, 2694-2703.

Crestaux, T., Le Maitre, O., Martinez, J. M., 2009. Polynomial Chaos Expansion for Sensitivity Analysis. Reliab. Eng. Sys. Safety. 94, 1161-1172.

Fisher, J., Bhattacharya, R., (2008). Stability analysis of stochastic systems using polynomial chaos. 2008 Amercan Control Conference. 4250-4255.

Fishman, G. S., 1996. Monte Carlo, Concepts, Algorithms and Applications, first Ed, Springer Verlag.

Ghanem, R.G., Spanos, P. D., 1991. Stochastic Finite Elements: a Spectral Approach, revised Ed, Springer Verlag.

Ganapathysubramanian, B., Zabaras, N., 2007. Sparse grid collocation schemes for stochastic natural convection problems. J. Comput.Phys. 225, 652-685.

Helton, J. C., Davis, F. J., 2003. Latin hypercube sampling and the propagation of uncertainty in analyses of complex systems. Reliab. Eng. Sys. Safety. 81, 23-69.

Hover, F.S., Triantafyllou, M. S., 2006. Application of polynomial chaos in stability and control. Automatica. 42, $789-795$.

Hultèn. J, (1993). Drum break squeal- a self exciting mechanism with constant friction. In SAE truck and bus meeting,1993, Detroit, Mi, USA SAE paper 932965.

Hoffmann, N., Gaul, L., (2003). Effects of damping on mode-coupling instability in friction-induced oscillations. ZAMM Z. Angew. Math. Mech, 83, 524-534.

Ibrahim, R.A., 1994a. Friction-induced vibration, chatter, squeal and chaos: Part I- Mechanics of contact and friction. ASME Appl. Mech. Rev. 47, 209-226.

Ibrahim, R.A., 1994b. Friction-induced vibration, chatter, squeal and chaos: Part II- Dynamics and modeling. ASME Appl. Mech. Rev. 47, 227-253. 
Isukapalli, S. S., Roy, A., Georgopoulos, P. G., 1998. Stochastic response surface methods (SRSMs) for uncertainty propagation: application to environmental and biological systems. Risk Analysis. 18, 351-363.

Isukapalli, S. S., Roy, A., Georgopoulos, P. G., 1998. Development and application of methods for assessing uncertainty in photochemical air quality problems. Interim Report for U.S.EPA National Exposure Research Laboratory.

Jakerman, J. D., Roberts, S. G., 2009. Stochastic galerkin and collocation methods for quantifying uncertainties in differential equation: a review. ANZIAM J. 50, 815-830.

Le Maître, O. P., Knio, O. M., Najm, H. N., Ghanem, R. G., 2001. A stochastic projection method for fluid flow Basic formulation. J. Comput.Phys. 173, 481-511.

Li, J., Xiu, D., 2009. A generalized polynomial chaos based ensemble Kalman filter with high accuracy. J. Comput. Phys. 228, 5454-5469.

Lindsley. N. J., Beran. P. S, (2005). Increased efficiency in the stochastic interrogation of an uncertain nonlinear aeroelastic system. International Forum on Aeroelasticity and Structural Dynamics, Munich, Germany, IF-055, June, 2005

Millman. D.R, King, P. I, Beran. P.S., (2003). Personal communication. A stochastic approach for predicting bifurcation of a pitch and plung airfoil. In: 21st AIAA Applied Aerodynamics Conference, Orlando, FL, 2003 AIAA-2003-3515.

Nagy, Z. K., Braatz, R.D., 2006. Distributional uncertainty analysis using power series and polynomial chaos expansions. J. Process Control. 17, 229-240.

Papadrakakis, M., Papadopoulos, V., 1999. Parallel solution methods for stochastic finite element analysis using Monte Carlo simulation. Comput. Methods Appl. Mech. Engrg. 168, 305-320. Prajna, S., Papachristodoulou, A., Seiler, P., Parrilo, P. A., (2005). SOSTOOLS and its Control applications, in: Henrion, D., Garulli, Andrea., Positive Polynomial in Control. Springer, pp. 273-292. Pettit, C.L., Beran, P.S., 2006. Spectral and multiresolution Wiener expansions of oscillatory stochastic process. J. Sound Vib. 294, 752-779.

Ragot, P., Berger, S., Sinou, J.J., Aubry, E., 2008. Interval approach applied to blades of windscreens wiper. Int. J. Pure Appl. Math. 46, 643-648. 
Saad, G., Ghanem, R., Masri, S., (2007). Robust system identification of strongly non-linear dynamics using a polynomial chaos based sequential data assimilation technique. Structural Dynamics and Materials Conference, Honolulu, USA.

Sandu, A., Sandu, C., Ahmadian, M., 2006. Modeling multibody dynamic systems with uncertainties. Part I: Numerical application. Multibody system Dynamic. 15, 369-391.

Sandu, C., Sandu, A., Ahmadian, M., 2006. Modeling multibody dynamic systems with uncertainties. Part II: Theoretical and computational aspects. Multibody system Dynamic. 15, 241-262.

Shin, K., Oh, J.E., Brennan, M.J., 2002a. Nonlinear analysis of friction induced vibrations of a two degree of freedom model for disc brake squeal noise. JSME int. J. 45, 426-432.

Shin, K., Brennan, M. J., Oh, J-E., Harris, C. J., (2002b). Analysis of disc brake noise using a twodegree-of-freedom model. J.Sound Vib. 254, 837-848.

Sinou, J. J., Thouverez, F., Jezequel, L., 2004. Stability non-linear analysis of a complex rotor/stator contact. J. Sound Vib. 278, 1095-1129.

Sinou, J. J., Dereure, O., Mazet, F., Thouverez, F., Jezequel, L., 2006a. Friction-induced vibration for an aircraft brake system-Part 1: Experimental approach and stability analysis. Int. J. Mech. Sciences. $48,536-554$.

Sinou, J. J., Thouverez, F., Jezequel, L., Dereure. O., Mazet, F, 2006b. Friction-induced vibration for an aircraft brake system-Part 2: Non-linear dynamics. Int. J. Mech. Sciences. 48, 555-567.

Sinou, J. J., Jezequel, L., 2007. Mode coupling instability in friction-induced vibrations and its dependency on system parameters including damping. Eur. J. Mech A/Solid, 26, 107-122.

Slotine. J. J E., 1991. Applied nonlinear control, Prentice-Hall Inc.

Smith, A. H. C., Monti, A., Ponci, F., 2007. Indirect measurements via a polynomial chaos observer. IEEE Trans. Instrum. Meas. 56, 743-752.

Sudret, B., 2007. Global sensitivity analysis using polynomial chaos expansions. Reliab. Eng. Sys. Safety. 93, 964-979.

Wan, X., Karniadakis, G., 2006. Beyond Wiener-Askey expansions: handling arbitrary PDFs. J. Scientific Comp. 27, 455-464.

Wiener, N., 1938. The homogeneous chaos. Am J. Math. 60, 897-936. 
Williams, M. M. R., 2006. Polynomial chaos functions and stochastic differential equations. Annals of Nuclear Energy. 33, 774-785.

Witteveen, J.A.S., Loeven, A., Sarkar, S., Bijl, H., 2007. Probabilistic collocation for period-1 limit cycle oscillations. J. Sound Vib. 311, 421-439.

$\underline{\text { Xiu, D., Luckor, D., Su, C-H., Karnadakis, G. E., 2003. Performance Evaluation of Generalized }}$ Polynomial Chaos. Lecture Notes in Computer Science, Springer Berlin.

Xiu, D., Karniadakis, G. E., 2003. Modelling uncertainty in flow simulations via generalized polynomial chaos. J. of Comp. Physics. 187, 137-167.

Xiu, D., Karniadakis, G. E., 2002. Modeling uncertainty in steady state diffusion problems via generalized polynomial chaos, Comput. Methods Appl. Mech. Eng. 191, 4927-4948.

\begin{tabular}{|l|l|l|l|l|l|l|l|}
\hline & MC & Intrusive PC & Error (\%) & Collocation & Error (\%) & Regression & Error (\%) \\
\hline Global min $\left(\mathrm{X}_{1}\right)$ & -0.3309 & -0.3444 & 4.08 & -0.3347 & 1.15 & -0.3359 & 1.51 \\
\hline Global max $\left(\mathrm{X}_{1}\right)$ & 0.3309 & 0.3440 & 3.96 & 0.3353 & 1.13 & 0.3363 & 1.63 \\
\hline Global min(dX $/ \mathrm{dt})$ & -180.9520 & -188.6896 & 4.28 & -181.9281 & 0.54 & -183.6523 & 1.49 \\
\hline Global max $(\mathrm{dX} 1 / \mathrm{dt})$ & 180.9983 & 188.8801 & 4.35 & 182.1842 & 0.66 & 183.8365 & 1.57 \\
\hline
\end{tabular}

Tab.1. Relative errors, MC solutions as reference results with $P=20$

\begin{tabular}{|l|l|l|l|l|l|}
\hline & MC & Collocation & Error (\%) & Regression & Error (\%) \\
\hline Global $\min \left(\mathrm{X}_{1}\right)$ & -0.3309 & -0.3307 & 0.06 & -0.3309 & 0.00 \\
\hline Global $\max \left(\mathrm{X}_{1}\right)$ & 0.3309 & 0.3307 & 0.06 & 0.3309 & 0.00 \\
\hline Global $\min \left(\mathrm{dX}_{1} / \mathrm{dt}\right)$ & -180.9520 & -181.9158 & 0.53 & -181.2867 & 0.18 \\
\hline Global $\max (\mathrm{dX} 1 / \mathrm{dt})$ & 180.9983 & 181.8950 & 0.50 & 181.3285 & 0.21 \\
\hline
\end{tabular}

Tab.2. Relative errors, $\mathrm{MC}$ solutions as reference results with $P=30$ 


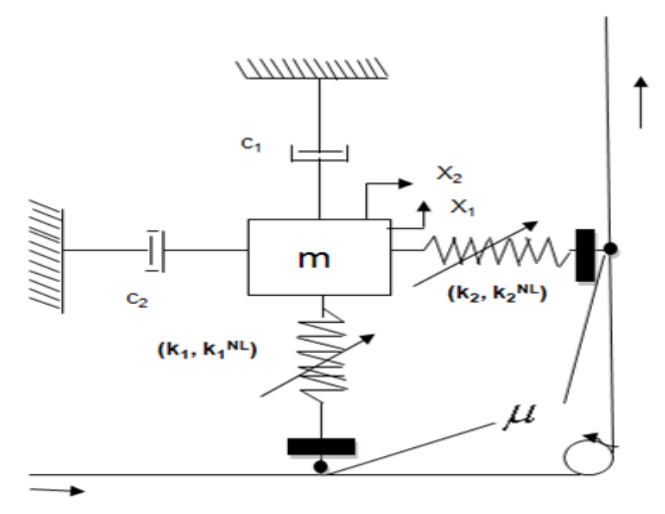

Fig.1. Mechanical model
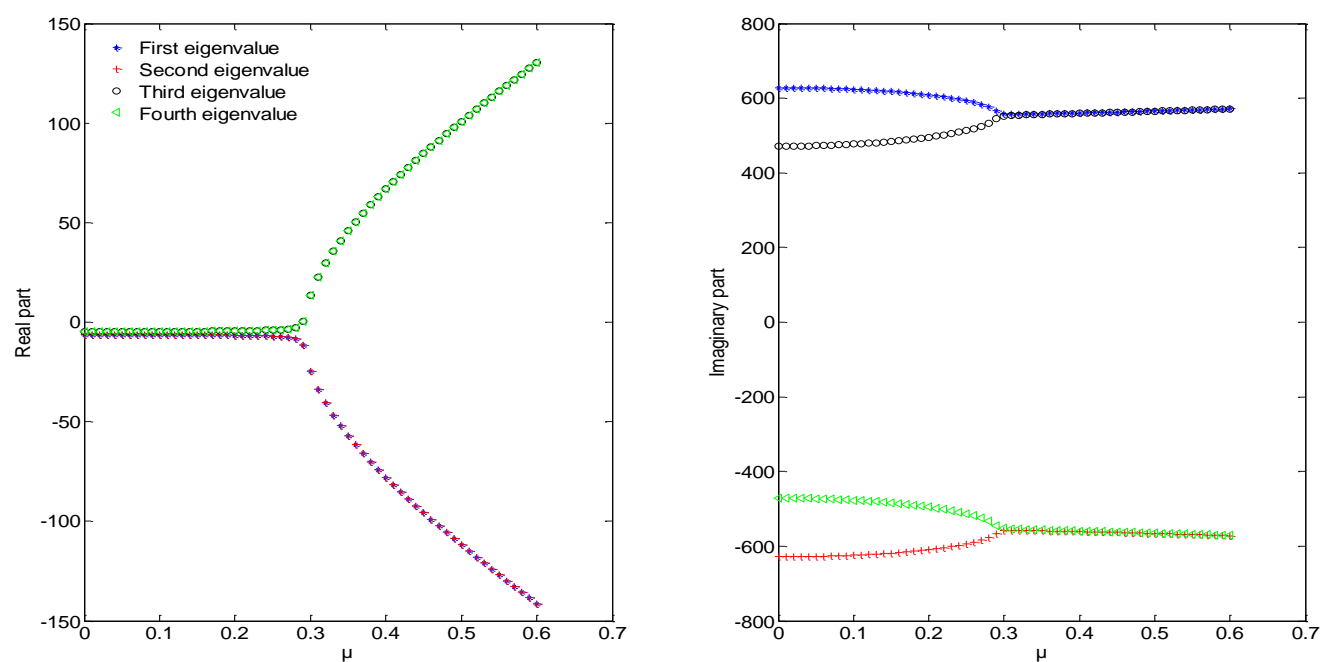

Fig.2. Evolution of the real part of eigenvalues according to the friction coefficient values 

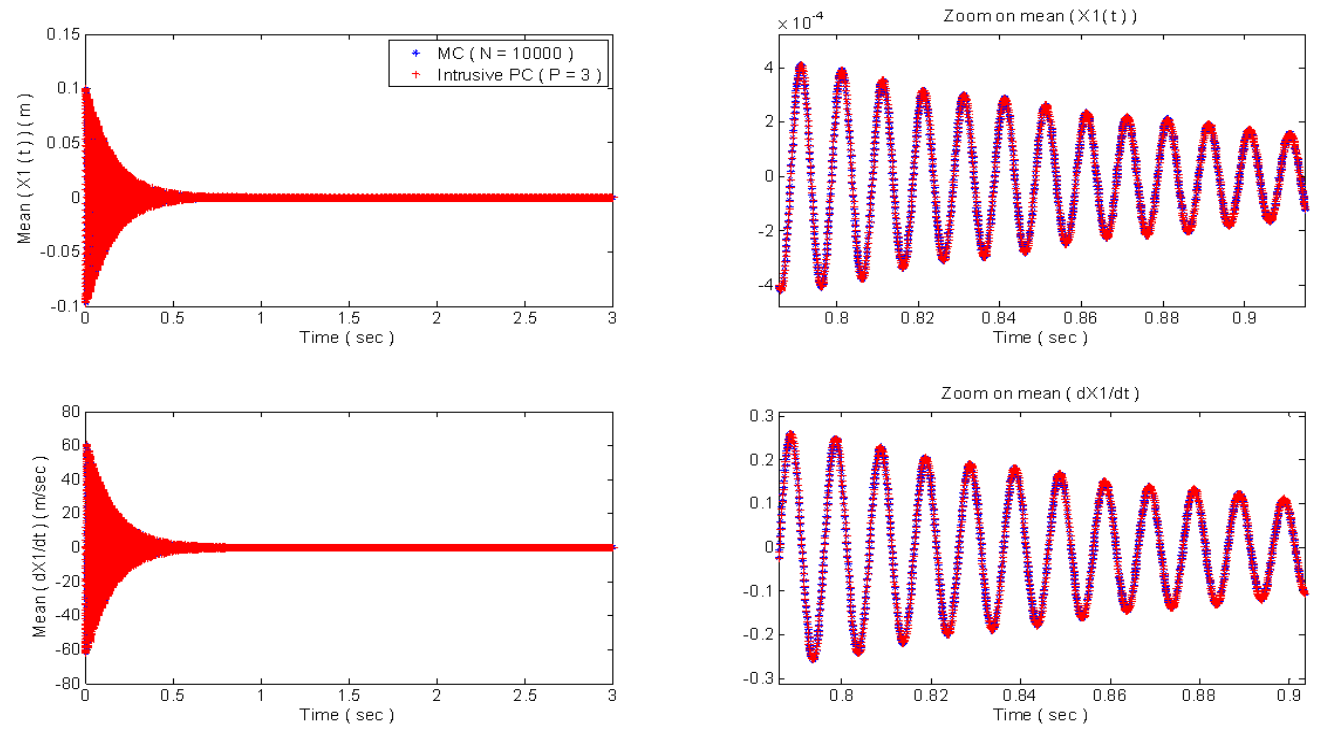

Fig.3. Instantaneous mean value $X_{1}(t)$ and $\dot{X}_{1}(t)$
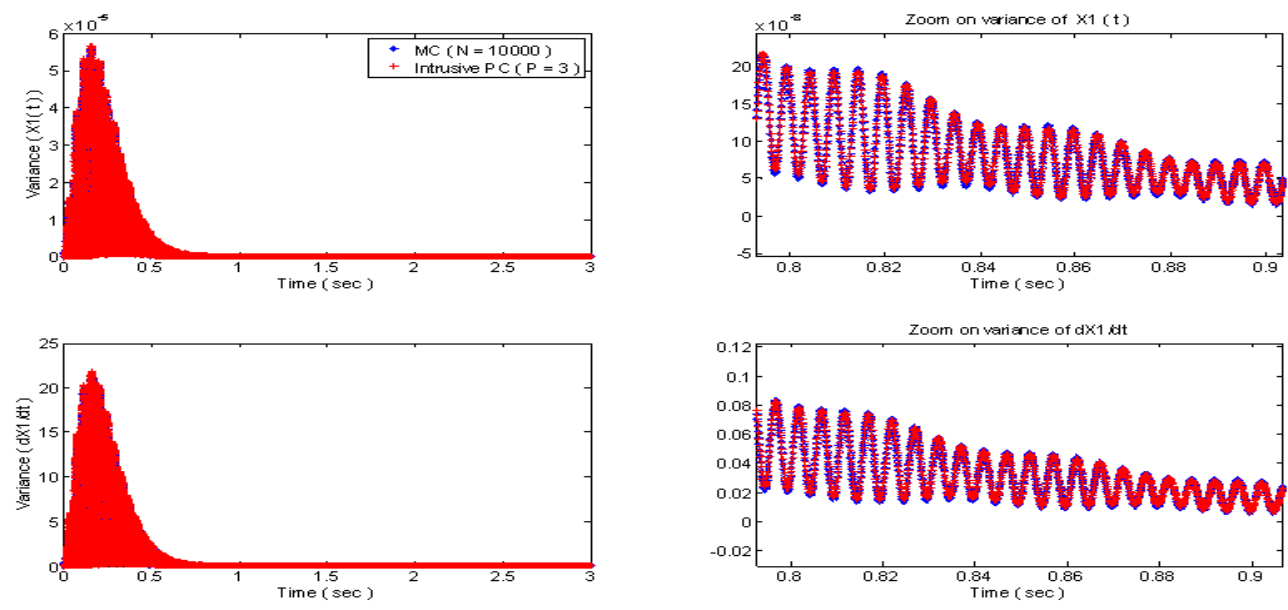

Fig.4. Instantaneous variance of $X_{1}(t)$ and $\dot{X}_{1}(t)$ 

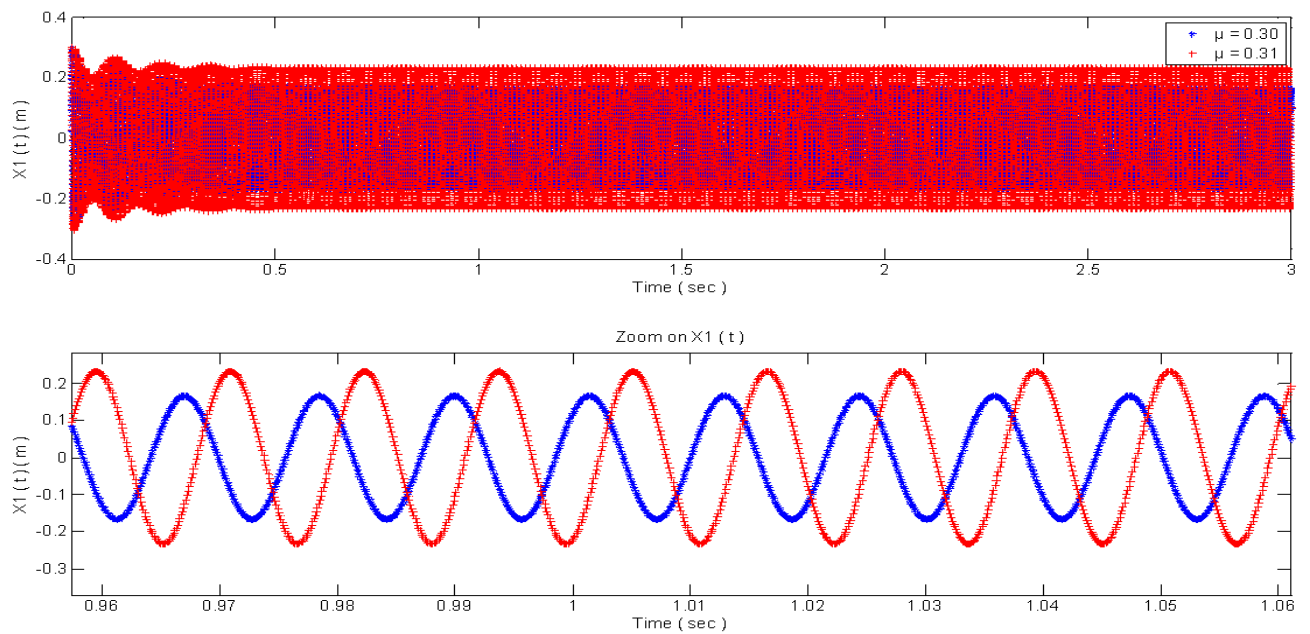

Fig.5. Instantaneous displacement $X_{1}(t)$ corresponding to two values of the friction coefficient

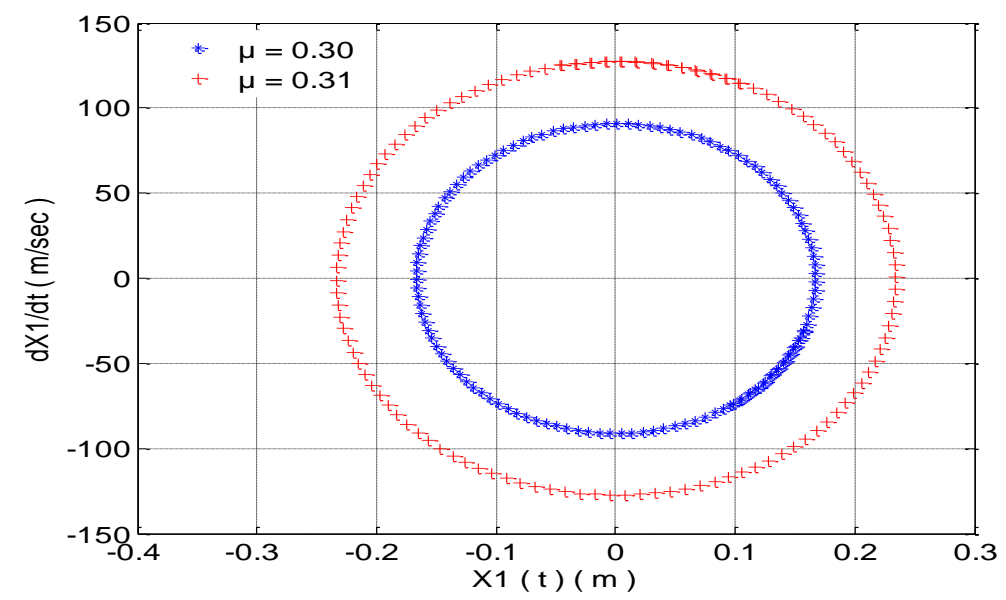

Fig.6. Limit cycle corresponding to two values of the friction coefficient
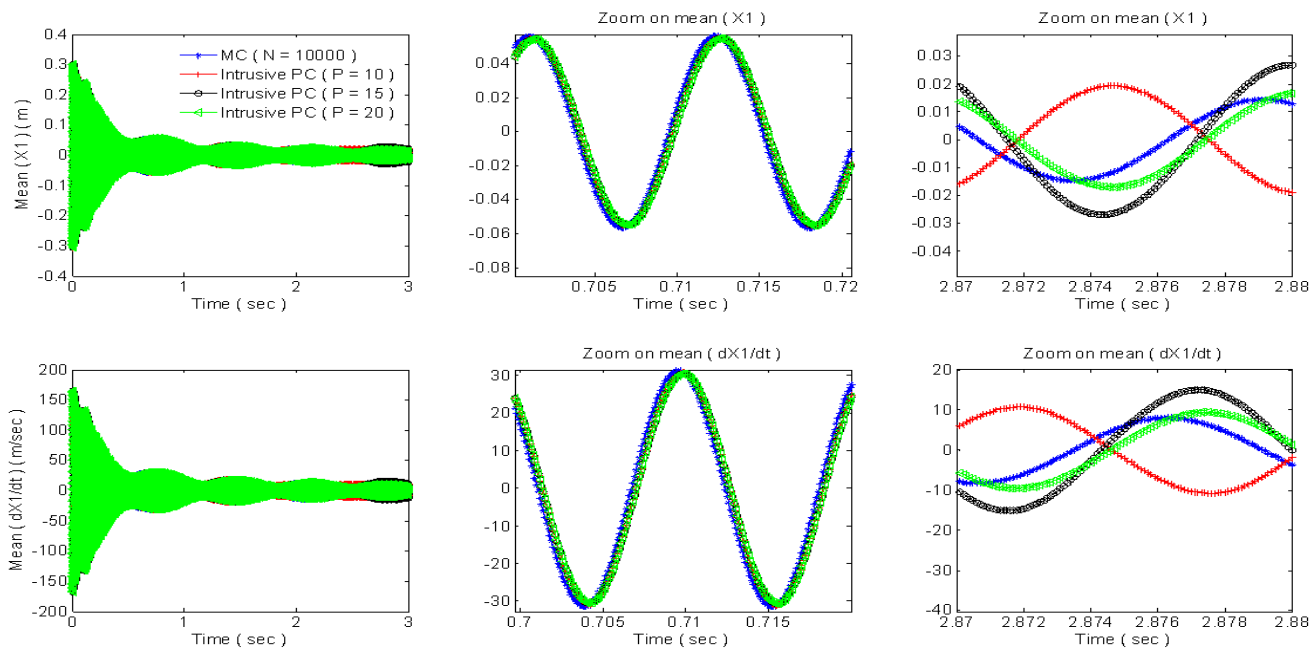
Fig.7. Instantaneous mean value of $X_{1}(t)$ and $\dot{X}_{1}(t)$
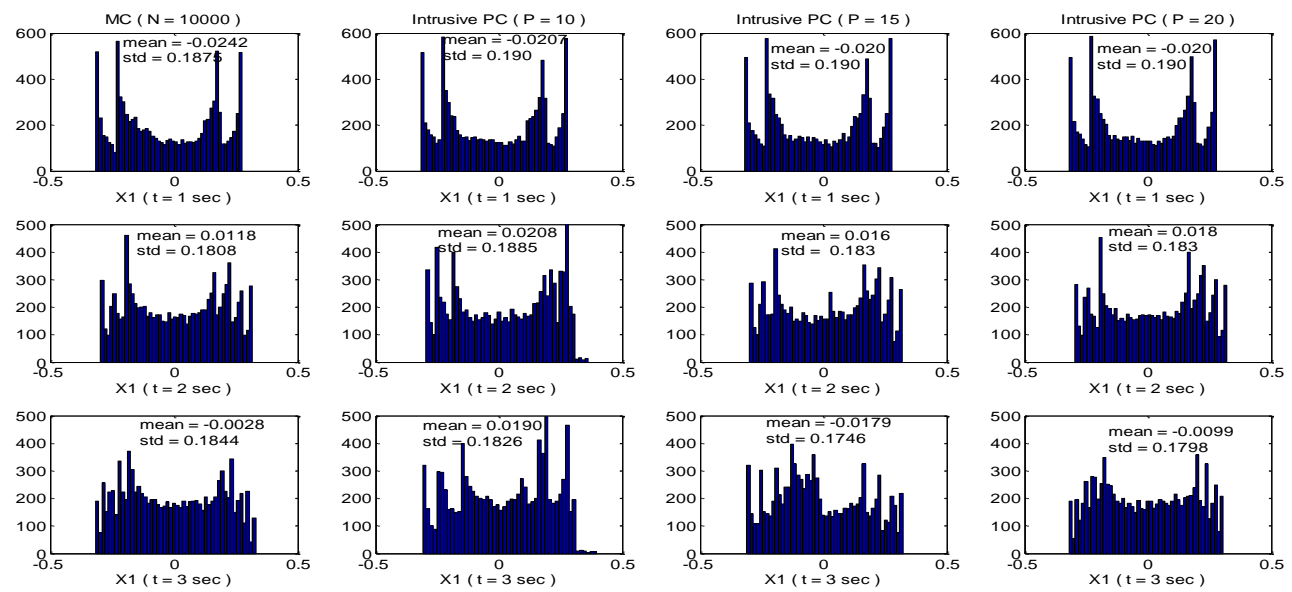

Fig.8. Distribution of $X_{1}(t)$ at $t=1 s, t=2 s$ and $t=3 s$
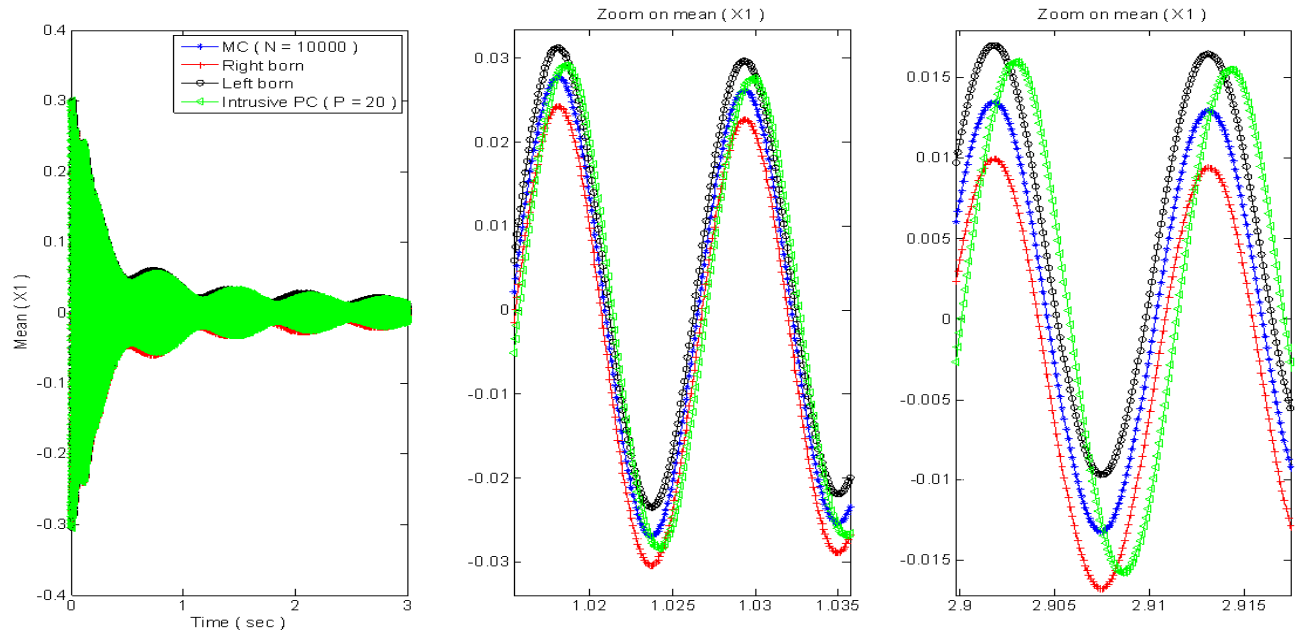

Fig.9. 95\% confidence interval for the instantaneous mean value of $X_{1}(t)$
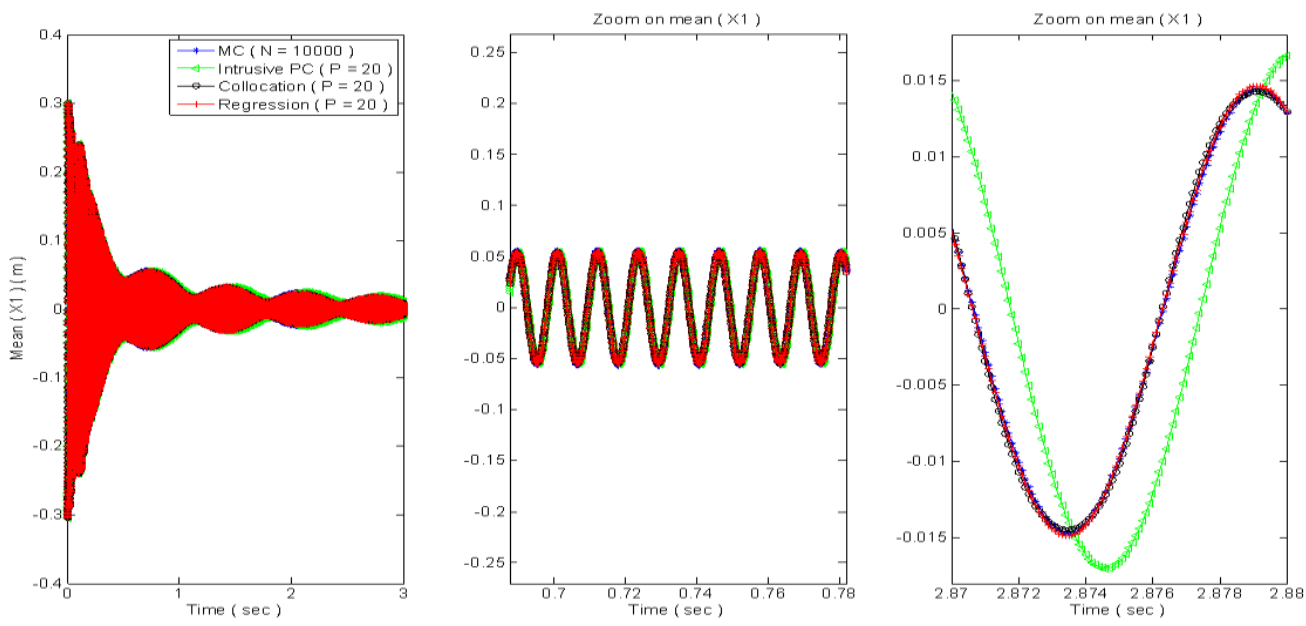
Fig.10. Instantaneous mean value of displacement $X_{1}(t)$
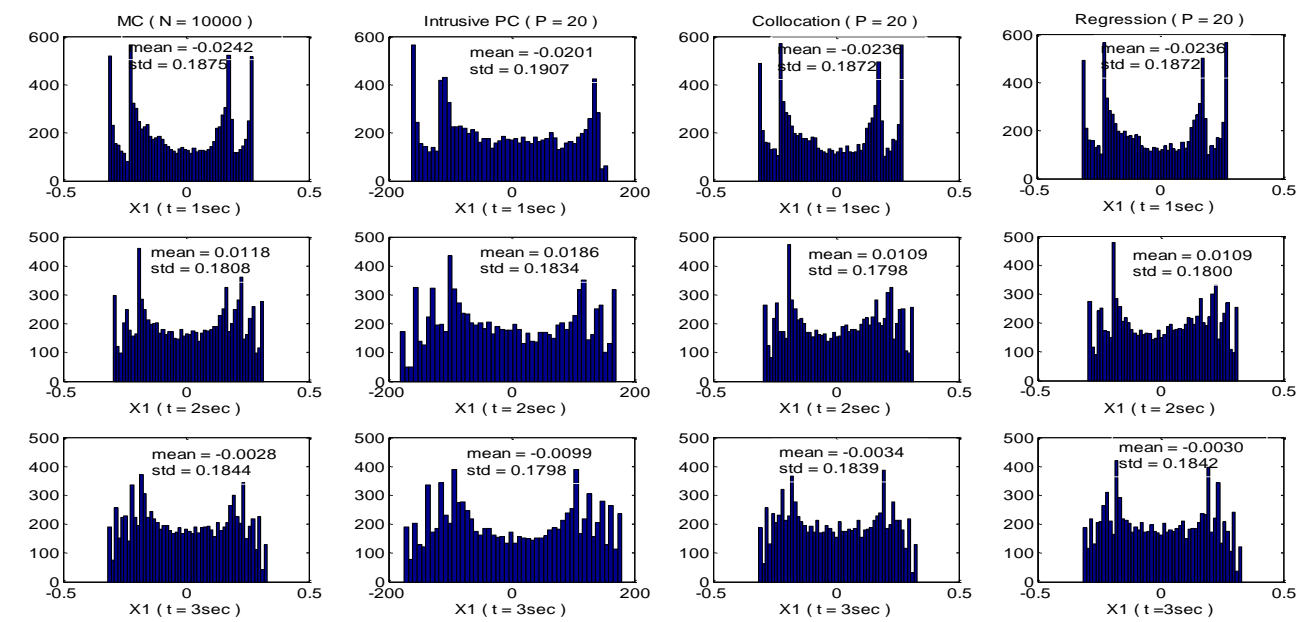

Fig.11. Distribution of $X_{1}(t)$ at $t=1 s, t=2 s$ and $t=3 s$
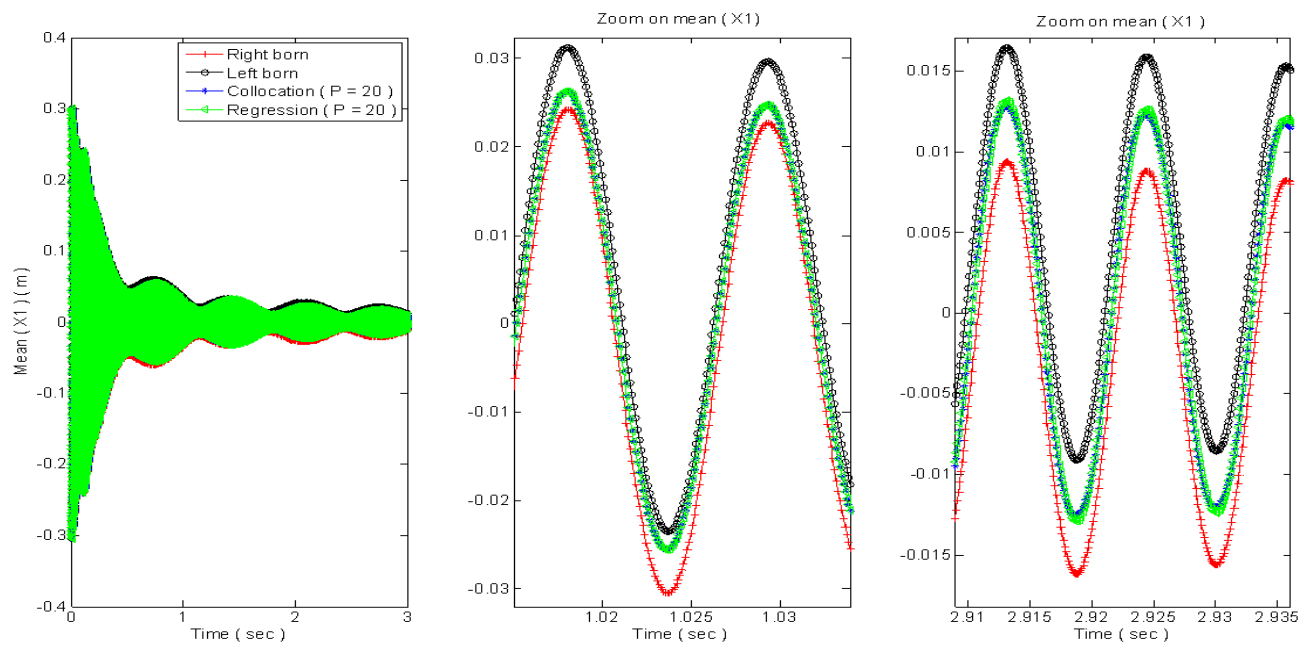

Fig. 12. 95\% confidence interval for the instantaneous mean value of $X_{1}(t)$ 